\title{
INDOOR ROUTE PLANNING UNDER HEXAGON NETWORK CONSIDERING MULTI-CONSTRAINS
}

\author{
Weicai Wang ${ }^{1}$, Tinghua $\mathrm{Ai}^{1 *}$,Chongya Gong ${ }^{1}$ \\ ${ }^{1}$ School of Resource and Environmental Sciences, Wuhan University, Wuhan, China - (weicai_wang, tinghuaai)@whu.edu.cn; \\ 334628177@qq.com
}

Commission IV, WG IV/5

KEY WORDS: Indoor Route Planning; Regular Hexagon; Multi-restrictions; A* Algorithm

\begin{abstract}
:
Indoor route planning, affected by many constraints, needs to take consideration of both the spatial geometry, environmental attribute information of the scene and the application preferences. Thus, it requires a data model that can integrate multiple constraints to model the indoor scene; meanwhile, it's also necessary to take into account the isotropic features of the indoor pathfinding behavior and the superposition analysis of multi-constraint conditions by the route-finding algorithm. Based on this, this paper proposes a multi-factor constrained $\mathrm{A}^{*}$ algorithm model based on the hexagonal grid, that is using an isotropic regular hexagon to model an indoor scene, and taking constraint conditions as heuristic factors to guide the route-finding algorithm so as to get the route. Based on this model, distance, recognition and pedestrian density are used as examples to illustrate the impact of constraints on route planning and their organic combination with scene modeling and route-finding algorithms. The experimental results show that this scheme can effectively take into account the constraints such as distance, landmark strength, and pedestrian heat, thus providing a route that is more in line with the application preferences.
\end{abstract}

\section{INTRODUCTION}

Indoor scenes are usually complex, so the indoor route planning is limited by multiple constraints. Taking into account a variety of constraints need to rely on flexible and efficient scene modeling. The current scene modeling model mainly includes models based on symbols, represented by the graph model, and those based on geometry, represented by the grid model (Afyouni I et al, 2012). In an indoor scene, the change of constraints is continuous rather than balanced, such as the influence of landmarks, changes in lights, etc. Therefore, the graph model is not conducive to the analysis and calculations of the integrated and overlaying constraints. While the grid model, using regular elements (equilateral triangles, squares, regular hexagons, etc.) to partition the interior space, can achieve a more elaborate representation of the scene, what's more, each cell can effectively integrate multiple constraints and therefore, effectively avoid the drawbacks of the graph model. In addition, comparing to the outdoor route-finding behavior, the indoor route-finding behavior is arbitrary in directions, that is, it is isotropic. However, due to the presence of common and diagonal edges, a square can cause inconsistencies of the neighborhood, so the regular hexagons of isotropy (Dieudonné Tsatcha et al, 2014) are more suitable for indoor scene modeling and route planning. As a heuristic search algorithm, the $A^{*}$ algorithm can set heuristic factors, narrow the search scope to improve search efficiency (Aine S et al ,2014), and can well take into account a variety of constraints, so it is suitable for the search of the optimal route. Thus, this paper proposes a multi-factor constrained A* algorithm model based on hexagonal grid.

\section{INDOOR SCENE MODELING}

The indoor structure is complex and the scene is diverse, so there are many constraints for the indoor route planning. This paper selects some constraints to conduct the scene modeling, including the constraints based on the scene itself, such as corridors, wall and other obstacles, and those based on application preferences, such as shop signs, indicator slogans as well as pedestrian distribution densities, etc.

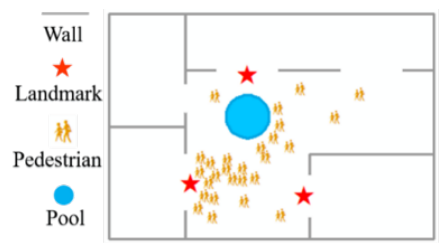

(a) Indoor scene vector

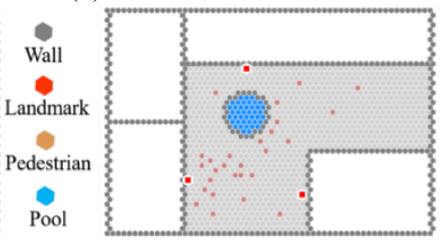

(b) Indoor scene hexagon

Figure 1. Indoor scene modeling

\subsection{Landmark Intensity}

In indoor navigation, up to $85 \%$ of the path descriptions involve landmarks (Liu Tao et al, 2017), what's more, the landmarks can help pedestrians better identify the direction and positioning, improve the visibility of the path, and increase the possibility of choosing a correct path. Different landmarks have different influence ranges and intensities due to differences in functionality, visibility, and significance. This paper proposes a model for the impact intensity, the formulas are as follows, and establishes the landmark intensity grid. 


$$
\begin{gathered}
S\left(l_{i}, g_{n}\right)=\left\{\begin{aligned}
S_{l_{i^{\prime}}} & 0 \leq D_{l_{i}, g_{n}} \leq R_{l_{i}} \\
S_{l_{i}}-S_{l_{l_{i}}} / R_{l_{i}} \cdot\left(D_{l_{i}, g_{n}}-R_{l_{i}}\right), & R_{l_{i}}<D_{l_{i}, g_{n}} \leq 2 R_{l_{i}} \\
0, & D_{l_{i}, g_{n}}>2 R_{l_{i}}
\end{aligned}\right. \\
S\left(g_{n}\right)=\sum_{i=0}^{N} S\left(l_{i}, g_{n}\right)
\end{gathered}
$$

$$
\begin{array}{ll}
\text { where } & \mathrm{l}=\text { landmark } \\
\mathrm{g}=\text { grid } \\
\mathrm{D}=\text { distance between two grids } \\
\mathrm{R}=\text { influence range of landmark } \\
\mathrm{S}=\text { landmark intensity }
\end{array}
$$

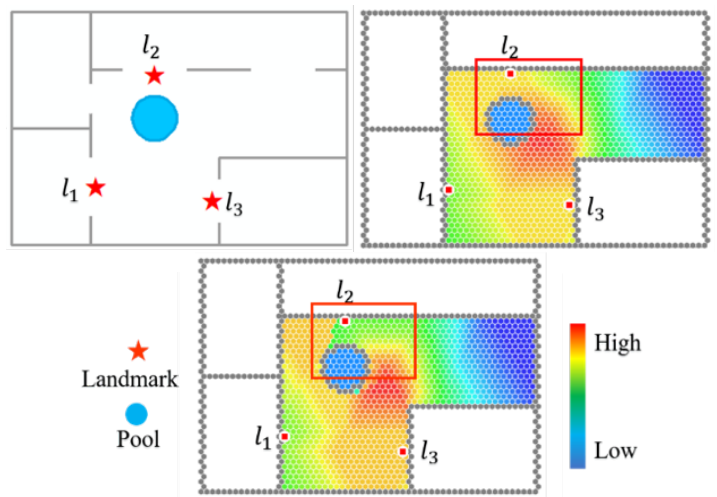

Figure 2. Landmark intensity grid

\subsection{Pedestrians Distribution Heat}

In route planning, the distribution of pedestrians influences the choice of routes, positively or negatively. For security personnel, the concentration of pedestrians which means the risk is a positive impact, so it needs to be approached. While for the passengers, it means congestion which is a negative impact and need to be avoided. Therefore, this paper uses Kernel Density Estimation algorithm(Yu Wenhao et al, 2015) to estimate pedestrians' distribution in continuous space based on their position information and to obtain the pedestrians distribution heat map.

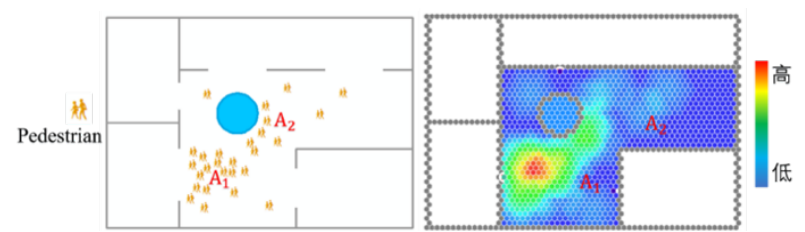

Figure 3. Pedestrian distribution heat grid

\section{A* ROUTE PLANNING ALGORITHM}

As a heuristic search algorithm, the $\mathrm{A}^{*}$ algorithm can set heuristic factors, narrow the search scope to improve search efficiency, and can well take into account a variety of constraints(Antikainen H, 2013), so it is suitable for the search of the optimal route. On the basis of the regular hexagonal scene modeling, the appropriate constraint conditions are selected, according to the specific application preferences, as the heuristic factor of the $\mathrm{A}^{*}$ algorithm, then the total estimated cost of the probe grid $g_{n}$ is the established cost plus the product of each heuristic factor and the proportion of the shares. The formula is as follows:

$$
F\left(g_{n}\right)=G\left(g_{n}\right)+\sum_{i}^{N} w_{i} \cdot H\left(g_{n}\right)_{i}
$$

where $G=$ actual cost

$\mathrm{H}=$ heuristic cost

$\mathrm{F}=$ estimated cost

In addition, the three enlightenment factors: distance, recognition, and pedestrian density selected in this paper, due to the inconsistent prime dimension, need to be normalized first(Zhao xinghao et al, 2005). The formula and detailed algorithm flow chart are as follows:

$$
\begin{gathered}
D\left(g_{n}, g_{D}\right)^{\prime}=\frac{D\left(g_{n}, g_{D}\right)}{D\left(g_{0}, g_{D}\right)} \\
S\left(l_{i}, g_{n}\right)^{\prime}=\left\{\begin{array}{l}
\frac{S\left(g_{n}\right)-S_{\min }}{S_{\max }-S_{\min }}, \text { Negative impact } \\
1-\frac{S\left(g_{n}\right)-S_{\min }}{S_{\max }-S_{\min }}, \text { Positive impact }
\end{array}\right. \\
K\left(g_{n}\right)^{\prime}=\frac{K\left(g_{n}\right)-K_{\min }}{K_{\max }-K_{\min }}
\end{gathered}
$$

where $\quad \mathrm{D}=$ distance between two grids $\mathrm{S}=$ landmark intensity $\mathrm{K}=$ pedestrian heat $\max =\max$ value $\min =\min$ value

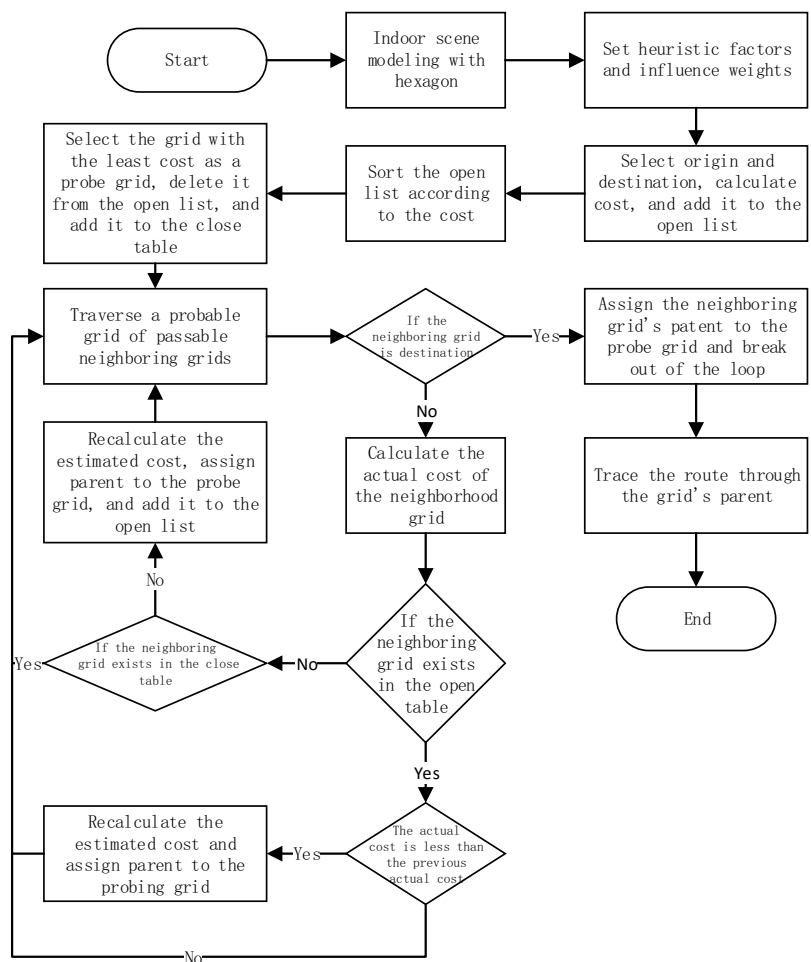

Figure 4. Flow chart of route planning based on A* algorithm

\section{EXPERIMENT}

\subsection{Experimental Configuration}

The experiment site is a shopping center and this paper selects three constraints: distance, recognition and pedestrian density, and configures five sets of weight coefficients, as shown in Table 1. The route planning results are denoted as $\mathrm{P}_{\mathrm{SPA}}, \mathrm{PD}$, $\mathrm{P}_{\mathrm{D}+\mathrm{S}}, \mathrm{P}_{\mathrm{D}+\mathrm{K}}$ and $\mathrm{P}_{\mathrm{D}+\mathrm{S}+\mathrm{K}}$. 


\begin{tabular}{|l|l|l|l|}
\hline Heuristic factors / Weight & $\mathrm{w}_{\mathrm{D}}$ & $\mathrm{w}_{\mathrm{S}}$ & $\mathrm{w}_{\mathrm{K}}$ \\
\hline Null(SPA) & 0 & 0 & 0 \\
$\mathrm{D}$ & 1 & 0 & 0 \\
$\mathrm{D}+\mathrm{S}$ & $1 / 2$ & $1 / 2$ & 0 \\
$\mathrm{D}+\mathrm{K}$ & $1 / 2$ & 0 & $1 / 2$ \\
$\mathrm{D}+\mathrm{S}+\mathrm{K}$ & $1 / 3$ & $1 / 3$ & $1 / 3$ \\
\hline
\end{tabular}

Table 1. Choice and weight configuration of heuristic factors

\subsection{Indoor Scene Modeling}

In order to discuss the difference between square and hexagon in indoor route planning, this experiment models the test regions through square and hexagon respectively, and creates a landmark intensity grid and a pedestrian thermal grid, as shown in Figure 5(a), (b). The experiment also selects two sets of ODs, takes into consideration the enlightenment factordistance, then obtains the route as shown in Figure 5(c)(d) below. By comparison, it can be found that the hexagon-based path is more in line with pedestrian path-seeking behavior, reflecting the advantage of hexagon: isotropy.

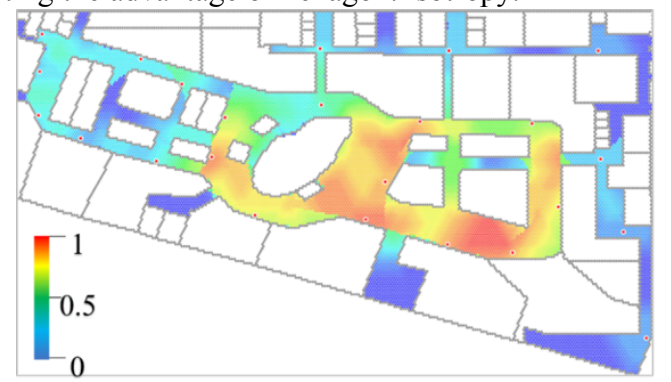

(a) Landmark intensity grid

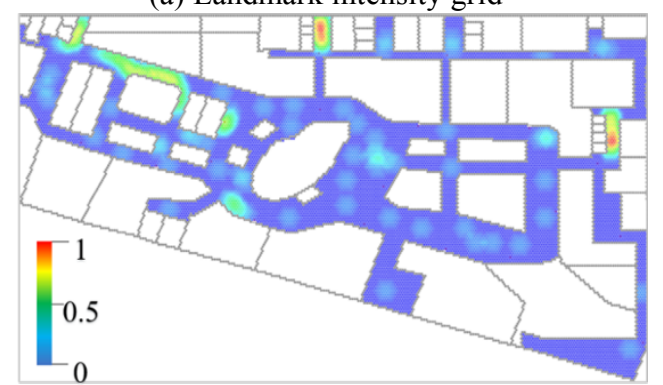

(b) Pedestrian heat grid

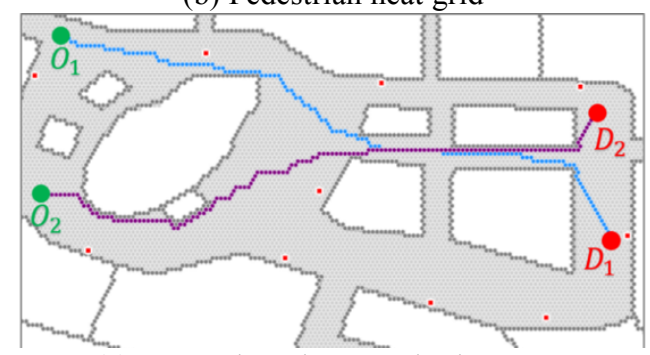

(c) Routes based on regular hexagon

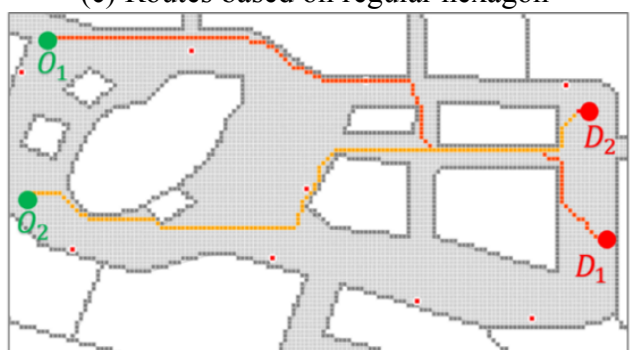

(d) Routes based on square

Figure 5. Landmark intensity, pedestrian heat grid, route planning results based on square and regular hexagon of experiment area

\subsection{Experimental Results}

In order to discuss the impact of multiple constraints on the indoor route planning and the effectiveness of the solution based on the hexagonal route planning, this paper selects four groups of ODs in the test regions, conducts five route plannings according to the weight configuration mentioned above, and shows the route result of $O_{l} D_{l}$, as shown in Figure 6. Comparing the five routes, it can be found that $\mathrm{P}_{\mathrm{D}+\mathrm{K}}$ and $\mathrm{P}_{\mathrm{D}+\mathrm{S}+\mathrm{K}}$ bypass region $A_{1}$, which indicates that taking into account the pedestrian's heat, the navigation can effectively avoid crowded areas; $\mathrm{P}_{\mathrm{D}+\mathrm{S}}, \mathrm{P}_{\mathrm{D}+\mathrm{K}}$ and $\mathrm{P}_{\mathrm{D}+\mathrm{S}+\mathrm{K}}$ all pass through region $A_{2}$, among which $\mathrm{P}_{\mathrm{D}+\mathrm{K}}$ and $\mathrm{P}_{\mathrm{D}+\mathrm{S}+\mathrm{K}}$, considering the pedestrian thermal factors and to avoid the crowded area $A_{1}$, reach $\mathrm{p}_{1}$, and their subsequent routes passing through the region $\mathrm{A}_{2}$ can also be explained from the prospect of distance, but $\mathrm{P}_{\mathrm{D}+\mathrm{S}}$ in the case of not avoiding the crowded area $\mathrm{A}_{1}$ to reach $\mathrm{p}_{2}$, still turns to the right and passes the region $\mathrm{A}_{2}$, indicating that the route taking into account the landmark intensity in the navigation can effectively guide pedestrians to areas with higher landmark intensity. Therefore, it can be seen that the proposed indoor route planning with multiple constraints is effective.

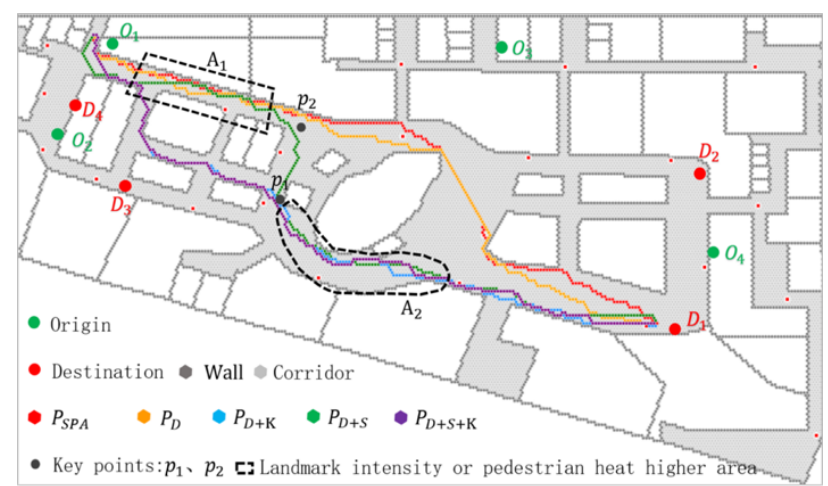

Figure 6. Results of indoor route planning

Meanwhile, in order to quantify the above-mentioned results, this paper selects such indicators as distance, average pedestrian heat, average landmark intensity, and the traversed grids to perform statistical analysis on different routes of the four groups of ODs. The results are shown as Figure 7(a)- (d).

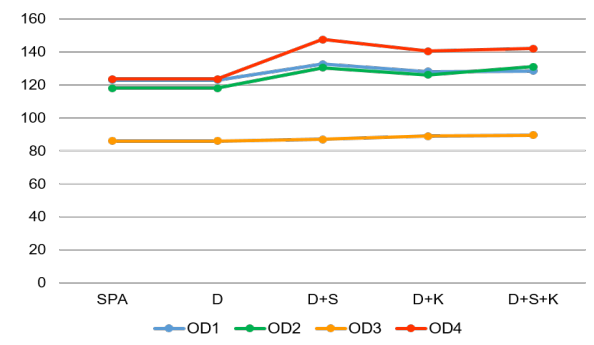

(a) Distance

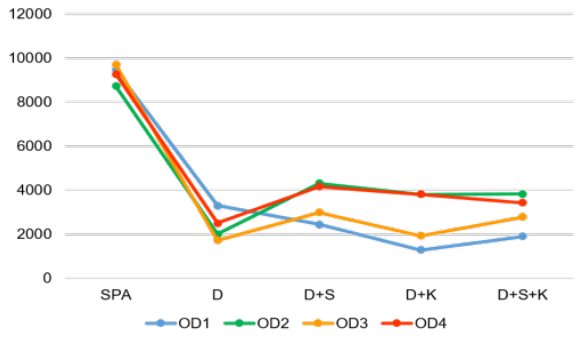

(b) Number of grids traversed 


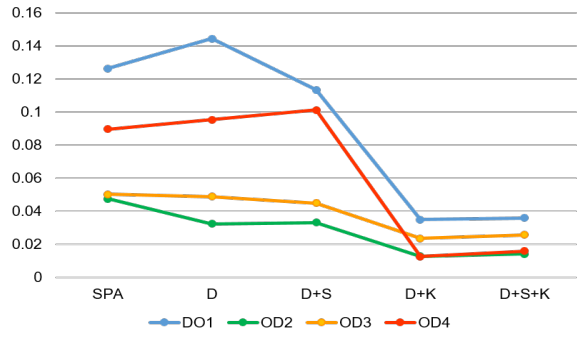

(c) Average pedestrian heat

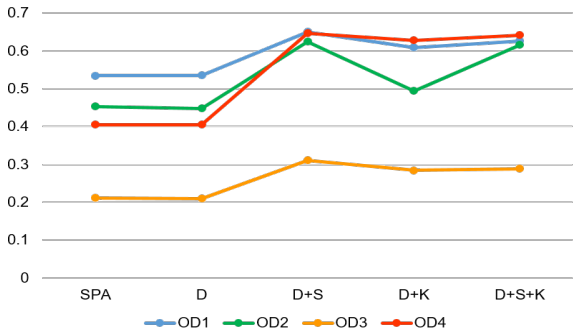

(d) Average landmark intensity

Figure 7. Compare of routes forming with different heuristic factor

\section{CONCLUSIONS}

This paper proposes an indoor scene modeling method based on hexagonal grid model, which can not only take into account various constraints, but also has the advantage of isotropy; by the $\mathrm{A}^{*}$ algorithm, it can effectively consider the influence of multiple enlightenment factors and obtains the route satisfying the application preference. The Experiment mentioned above shows that this method can effectively take into account various factors and provide a route that is more in line with application preferences. However, there are still deficiencies in the method which needs further improvement.

\section{REFERENCES}

Afyouni I, Ray C, Claramunt C. Spatial Models for Contextaware Indoor Navigation Systems: A Survey. In: Journal of Spatial Information Science, 2012, 1(4): 85-123.

Yang L, Worboys M. Generation of Navigation Graphs for Indoor Space. In: International Journal of Geographical Information Science, 2015, 29(10): 1737-1756.

Dieudonné Tsatcha, Éric Saux, Christophe Claramunt. A Bidirectional Path-finding Algorithm and Data Structure for Maritime Routing. In: International Journal of Geographical Information Science, 2014, 28(7): 1355-1377.

Aine S, Swaminathan S, Narayanan V, et al. Multi-Heuristic A*. In: International Journal of Robotics Research, 2014, 35(1-3): 224-243.

Liu Tao, Zhang Xing, Li Qingquan, etc al. An Indoor Pedestrian Route Planning Algorithm Base on Landmark Visibility. In: Geomatics and Information Science of Wuhan University, 2017, 42(1): 43-48.

Yu Wenhao, Ai Tinghua, Liu Pengcheng, etc al. Algorithm for Constructing Network Voronoi Diagram Based on Flow Extension Ideas. In: Acta Geodaetical et Cartographica Sinica, 2015, 44 (12): 1378-1383.
Antikainen H. Using the Hierarchical Pathfinding A* Algorithm in GIS to Find Paths through Rasters with Nonuniform Traversal Cost. In: ISPRS International Journal of Geo-Information, 2013, 2(4): 996-1014.

Zhao Xinghao, Deng Bing, Tao Ran. Dimensional Normalization in the Digital Computation of the Fractional Fourier Transform. In: Transactions of Beijing Institute of Technology, 2005, 25 (4): 360-364. 